\title{
PEANO SPACES WHICH ARE EITHER STRONGLY CYCLIC OR TWO-CYCLIC $\left({ }^{(}\right)$
}

\author{
BY \\ G. RALPH STROHL, JR.
}

1. Introduction. Let $M$ be a Peano space $\left({ }^{2}\right) p \in M$, and $\left\{p_{n}\right\}$ any infinite sequence of distinct points of $M$ converging to $p$. The space $M$ is said to be strongly arcwise connected at $p$ provided there is in $M$ a simple arc containing infinitely many of the points $\left\{p_{n}\right\}$, strongly cyclic at $p$ provided there is in $M$ a simple closed curve containing infinitely many of the points $\left\{p_{n}\right\}$, and two-cyclic at $p$ provided $M$ contains two arcs $\Gamma_{1}$ and $\Gamma_{2}$ such that each contains infinitely many of the points $\left\{p_{n}\right\}$ and $\Gamma_{1} \cap \Gamma_{2}=p$. If $M$ is strongly arcwise connected, strongly cyclic, or two-cyclic at each of its points, then $M$ is said to be strongly arcwise connected, strongly cyclic, or two-cyclic respectively. Strongly arcwise connected Peano spaces have been studied by Hall and Puckett $[1 ; 2]\left({ }^{3}\right)$.

It is clear that if $M$ is either strongly cyclic or two-cyclic at $p$, then $M$ is strongly arcwise connected at $p$; and that if $M$ is a cyclic Peano space which is two-cyclic at $p$, then $M$ is strongly cyclic at $p$. Moreover, it is easily shown that if $M$ is either strongly cyclic or two-cyclic at $p$, then $p$ lies in at least one, and at most a finite number, of true cyclic elements of $M$. Thus only cyclic Peano spaces will be considered.

An example of a cyclic Peano space which is strongly arcwise connected at a point $p$, but which fails to be strongly cyclic at $p$ follows. Let $M$ be the point set in the Euclidean plane consisting of the closed interval from 0 to 1 on the $x$-axis, the closed line segment of $y=x$ from $(0,0)$ to $(1,1)$, and the closed line segments from $(1 / n, 0)$ to $(1 / n, 1 / n)$ on the lines $x=1 / n, n \in I$. Considering the sequence of points $\left\{p_{n}\right\}$ where $p_{n}=(1 / n, 1 / 2 n), n \in I$, it is seen that $M$ fails to be strongly cyclic at the point $(0,0)$.

In this paper two characterizations of strongly cyclic Peano spaces will be obtained. The first will show that a cyclic Peano space which is strongly arcwise connected at $p$, but not strongly cyclic at $p$, is essentially like the example above. The second states that a cyclic Peano space $M$ is strongly cyclic if and only if for any infinite collection $\left\{V_{i}\right\}$ of open sets in $M$, there exists a simple closed curve in $M$ intersecting infinitely many of these sets.

For an example of a cyclic Peano space which is strongly cyclic at $p$, but

Presented to the Society October 22, 1955, under the title of Strongly cyclic peano spaces; received by the editors July $27,1956$.

(1) Presented to the Graduate School of the University of Maryland in partial fulfillment of the requirements for the degree of Doctor of Philosophy.

(2) All terms and symbols not explicitly defined in this paper may be found in [4] or [5].

(3) Numbers in brackers refer to the bibliography at the end of the paper. 
not two-cyclic at $p$, let $M$ denote the square in the Euclidean plane with vertices $(0,0),(1,0),(0,1)$, and $(1,1)$, either with or without its interior. Considering the sequence of points $(1 / n, 0), n \in I$, it is seen that $M$ is not twocyclic at the point $(0,0)$. If $M$ is considered without its interior, then $M$ fails to be two-cyclic at $(0,0)$ because this point lies on a free $\operatorname{arc}$ of $M$. If $M$ is considered with its interior, define in $M$ an arc $\Gamma$ consisting of $p$ and the union of the line segments of $x=1 / n$ from $(1 / n, 0)$ to $(1 / n, 1 / n)$, the line segments from $(1 / 2 n, 0)$ to $(1 / 2 n-1,0)$ on the $x$-axis, and the line segments from $(1 / 2 n+1,1 / 2 n+1)$ to $(1 / 2 n, 1 / 2 n)$ on the line $y=x, n \in I$. Thus given $\epsilon>0$, there exists an arc $\gamma \subset \Gamma$ such that $\gamma$ has $p$ as one endpoint, has diameter less than $\epsilon$, and $M-\gamma$ has an infinite collection of components converging to $p$.

It will also be shown that a cyclic Peano space which is strongly cyclic at $p$, but not two-cyclic at $p$, is essentially like the above example.

2. Lemmas. In this section several lemmas are given which will be useful in obtaining the principal results.

LEMma 2.1. If a limit point $p$ of an arcwise connected set $R$ is not regularly accessible from $R$, then there exists a positive number $\eta$ such that $R \cap S(p, \eta)$ has an infinite collection of distinct components $\left\{B_{i}\right\}$ such that $p \in \lim \inf \left\{B_{i}\right\}$. Moreover, $\bar{C} \cap F[S(p \eta),] \neq \varnothing$ for every component $C$ of $R \cap S(p, \eta)$.

Proof. This is a consequence of a lemma of E. E. Betz [3, p. 128].

LEMMA 2.2. Let $\alpha=q x p$ be an arc of a Peano space $M$ and $\left\{p_{n}\right\}$ an infinite sequence of distinct points of $M-\alpha$ converging to $p$. If, for every $\epsilon>0, S(p, \epsilon)$ contains an arc spanning $\alpha^{\prime}=\alpha-(q \cup p)$ and containing $a$ point of the sequence $\left\{p_{n}\right\}$, then $M$ contains an arc $\alpha^{*}$ from $q$ to $p$ containing infinitely many of the points $\left\{p_{n}\right\}$.

Lemma 2.3. Let $M$ be a cyclic Peano space and $J=\alpha \cup \beta$ a simple closed curve in $M$ where $\alpha=q x p$ and $\beta=q y p$ are arcs such that $\alpha \cap \beta=q \cup p, q \neq p$. Let $\left\{p_{n}\right\}$ be an infinite sequence of distinct points of $M-J$ converging to $p,\left\{x_{n}\right\}$ and $\left\{y_{n}\right\}$ infinite sequences of distinct points converging monotonically to $p$ on $\alpha$ and $\beta$ respectively. Assume that $M$ contains a collection $\left\{\lambda_{n}\right\}$ of distinct arcs converging to $p$ such that $\lambda_{n}$ is an arc $x_{n} p_{n} y_{n}$ spanning $J$, and that there is a positive number $\eta$ for which $(M-J) \cap S(p, \eta)$ has an infinite collection $\left\{B_{i}\right\}$ of components such that $\lambda_{i} \subset B_{i}$ and $\bar{B}_{i} \cap F[S(p, \eta)] \neq \varnothing$ for every $i$. Then there is a simple closed curve in $M$ containing infinitely many of the points $\left\{p_{n}\right\}$.

Proof. There is no loss of generality in assuming that the collection $\left\{B_{i}\right\}$ converges to a connected set $L$, and that $L$ contains a nondegenerate subarc $z p$ of $\alpha$. Let $\delta$ be a positive number such that $S(p, \delta) \cap \alpha \subset z p$. There exists a point $r \in S(p, \delta) \cap \alpha$ such that $r \neq x_{i}$ for all $i$. There exists an integer $n_{1}$, such that in the natural order from $q$ to $p$ on $\alpha, r<x_{n}$ for $n \geqq n_{1}$, and $x_{n_{1}-1}<r$. Let $U$ be a neighborhood of $r$ such that $\bar{U} \cap \beta=\varnothing, \bar{U} \subset S(p, \delta)$, and $\bar{U} \cap \alpha \subset x_{n_{1}-1} x_{n_{1}}$. There exists an integer $n_{2} \geqq n_{1}+2$ such that $B_{n_{2}} \cup s$ contains 
an arc stw where $s \in \bar{U} \cap \alpha$ and $w=\lambda_{n_{2}} \cap s t w$. Let $\Gamma_{1}$ be the union of the subarc $q s$ of $\alpha$, the arc stw, the subarc $w y_{n_{2}}$ of $\lambda_{n_{2}}$, and the subarc $y_{n_{2}} p$ of $\beta ; \Gamma_{2}$ the union of the subarc $q y_{n_{1}+1}$ of $\beta$, the arc $\lambda_{n_{1}+1}$, and the subarc $x_{n+1} p$ of $\alpha$. Then $\Gamma_{1} \cup \Gamma_{2}$ is a simple closed curve containing the point $p_{n_{1}+1}$.

Let $\delta_{2}$ be a positive number such that $\bar{S}\left(p, \delta_{2}\right) \cap\left(\Gamma_{1} \cup \Gamma_{2}\right)$ contains only points of the subarcs $x_{n_{2}} p$ and $y_{n_{2}} p$ of $\alpha$ and $\beta$ respectively. The above construction may be repeated in $S\left(p, \delta_{2}\right)$, thereby yielding a simple closed curve containing a second point of the sequence $\left\{p_{n}\right\}$. The proof of the lemma follows by continuing this process inductively.

Lemma 2.4. Let $M$ be a Peano space, $p \in M, \alpha=q x p$ an arc of $M$, and $\left\{p_{n}\right\}$ an infinite sequence of distinct points of $M$ converging monotonically to $p$ on $\alpha$. Assume there is a positive number $\eta$ and an infinite collection $\left\{D_{i}\right\}$ of components of $(M-\alpha) \cap S(p, \eta)$ such that (a) $p \in \lim \inf \left\{D_{i}\right\} ;$ (b) $\bar{D}_{i} \cap F[S(p, \eta)]$ $\neq \varnothing$ for every $i$. Then there exist arcs $\Gamma_{1}$ and $\Gamma_{2}$ in $M$ each of which contains infinitely many of the points $\left\{p_{n}\right\}$ and such that $\Gamma_{1} \cap \Gamma_{2}=p$.

Proof. There is no loss of generality in assuming that the collection $\left\{D_{i}\right\}$ converges to a connected set $L$, and that $L$ contains a nondegenerate subarc $z p$ of $\alpha$. Let $\sigma_{1}$ be a positive number such that $\bar{S}\left(p, \sigma_{1}\right) \cap \alpha \subset z p$. In the natural order from $q$ to $p$ on $\alpha$ let $t_{1}$ be the last point of $\alpha \cap\left[\bar{S}\left(p, \sigma_{1}\right)-S\left(p, \sigma_{1}\right)\right]$, and $p_{1}^{*}$ the first point of the sequence $\left\{p_{n}\right\}$ such that $t_{1}<p_{1}^{*}$. Let $\sigma_{2}$ be a positive number such that $\sigma_{2}<\sigma_{1} / 2$ and $\bar{S}\left(p, \sigma_{2}\right) \cap \alpha$ contains only points of $\alpha$ which follow $p_{1}^{*}$ in the natural order from $q$ to $p$ on $\alpha$. In the natural order from $q$ to $p$ on $\alpha$ let $t_{2}$ be the last point of $\alpha \cap\left[\bar{S}\left(p, \sigma_{2}\right)-S\left(p, \sigma_{2}\right)\right]$, and $p_{2}^{*}$ the first point of the sequence $\left\{p_{n}\right\}$ such that $t_{2}<p_{2}^{*}$. Continuing this process inductively yields sequences $\left\{\sigma_{i}\right\},\left\{t_{i}\right\}$, and $\left\{p_{i}^{*}\right\}$ with the following properties:

(i) $\left\{\sigma_{i}\right\}$ is a null sequence of positive numbers such that $\sigma_{i+1}<\sigma_{i} / 2$, and $\bar{S}\left(p, \sigma_{i+1}\right) \cap \alpha$ contains only points of $\alpha$ which follow $p_{i}^{*}$ in the natural order from $q$ to $p$ on $\alpha$.

(ii) $\left\{p_{i}^{*}\right\}$ is a subsequence of the $\left\{p_{i}\right\}$ such that $p_{i}^{*} \in S\left(p, \sigma_{i}\right)-\bar{S}\left(p, \sigma_{i+1}\right)$.

(iii) $t_{i}$ is the last point of $\alpha \cap\left[\bar{S}\left(p, \sigma_{i}\right)-S\left(p, \sigma_{i}\right)\right]$ in the natural order from $q$ to $p$ on $\alpha$.

(iv) In the natural order from $q$ to $p$ on $\alpha, t_{i}<p_{i}^{*}<t_{i+1}, i \in I$.

Let $\left\{V_{i}\right\}$ be a null sequence of regions such that $t_{i} \in V_{i}$ and $\bar{V}_{i} \cap \alpha$ contains only points interior to the subarc $p_{i-1}^{*} p_{i}^{*}$ of $\alpha$. Then there exists a collection of distinct arcs $\left\{e_{i} f_{i+3}\right\}$ spanning $\alpha$ and such that $e_{i} f_{i+3}$ lies in $V_{i} \cup\left[\bar{S}\left(p, \sigma_{i}\right)-S\left(p, \sigma_{i+3}\right)\right] \cup V_{i+3}$. The proof of the lemma is completed by defining the arcs $\Gamma_{1}$ and $\Gamma_{2}$ as follows:

$$
\begin{aligned}
& \Gamma_{1}=p \cup\left[\bigcup_{n \in I}\left(e_{4 n-3} f_{4 n}\right)\right] \cup\left[\bigcup_{n \in I}\left(f_{4 n} p_{4 n}^{*} e_{4 n+1}\right)\right] \\
& \Gamma_{2}=p \cup\left[\bigcup_{n \in I}\left(e_{4 n-1} f_{4 n+2}\right)\right] \cup\left[\bigcup_{n \in I}\left(f_{4 n+2} p_{4 n+2}^{*} e_{4 n+3}\right)\right]
\end{aligned}
$$


where the collections $\left\{f_{4 n} p_{4 n}^{*} e_{4 n+1}\right\}$ and $\left\{f_{4 n+2} p_{4 n+2}^{*} e_{4 n+3}\right\}$ are subarcs of $\alpha$.

3 . Separation theorem. In this section the following theorem is proved.

TheORem 3.1. Let $M$ be a cyclic Peano space which is strongly arcwise connected at $p$ but not strongly cyclic at $p$, and let $J$ be any simple closed curve in $M$ containing $p$. Let $J=\alpha \cup \beta$ where $\alpha=q x p$ and $\beta=q y p$ are arcs such that $\alpha \cap \beta$ $=q \cup p, q \neq p$. Then there exist in $M$ an infinite sequence of distinct points $\left\{p_{n}\right\}$, and a sequence of simple arcs $\left\{\lambda_{n}\right\}$ having the following properties:

(i) $p_{n} \in M-J$ for every $n$.

(ii) $\left\{p_{n}\right\}$ converges to $p$.

(iii) No simple closed curve in $M$ contains infinitely many points of the sequence $\left\{p_{n}\right\}$.

(iv) Every simple arc in $M$ containing infinitely many points of the sequence $\left\{p_{n}\right\}$ intersects each of the arcs $\alpha$ and $\beta$ in a set of points having $p$ as a limit point.

(v) Each $\lambda_{n}$ is an arc $x_{n} y_{n}$ spanning $J$ and containing the point $p_{n}$.

(vi) $\lambda_{m} \cap \lambda_{n}=\varnothing$ for $m \neq n$.

(vii) $\left\{\lambda_{n}\right\}$ converges to $p$.

(viii) $\cup_{n \in I} x_{n} \subset \alpha^{\prime}=\alpha-(q \cup p), \cup_{n \in I} y_{n} \subset \beta^{\prime}=\beta-(q \cup p)$.

(ix) The sequences $\left\{x_{n}\right\}$ and $\left\{y_{n}\right\}$ converge monotonically to $p$ on the arcs $\alpha$ and $\beta$ respectively.

(x) For $m \neq n$, the sets $\lambda_{m}^{\prime}=\lambda_{m}-\left(x_{m} \cup y_{m}\right)$ and $\lambda_{n}^{\prime}=\lambda_{n}-\left(x_{n} \cup y_{n}\right)$ lie in different components of $M-J$. Furthermore, these components converge to $p$. Thus for any simple closed curve $J$ of $M$ containing $p$, every arc of $J$ having $p$ as an interior point separates $M$ into infinitely many distinct components.

(xi) If $\left\{R_{i}\right\}$ is the collection of components of $M-J$ such that $\lambda_{i}^{\prime} \subset R_{i}$, then for no value of $i$ is there an arc of $\bar{R}_{i}-\beta$ spanning $\alpha$ and containing the point $p_{i}$, or an arc of $\bar{R}_{i}-\alpha$ spanning $\beta$ and containing the point $p_{i}$.

Proof. Since $M$ is not strongly cyclic at $p$, there exists in $M$ an infinite sequence of distinct points $\left\{p_{n}\right\}$ satisfying (ii) and (iii). From (iii) it follows that $J$ contains at most a finite number of the points $\left\{p_{n}\right\}$. Hence no generality is lost in assuming that (i) holds.

Since $M$ is strongly arcwise connected at $p$, there exists in $M$ an arc containing infinitely many of the points $\left\{p_{n}\right\}$. Let $\lambda$ be an arbitrary arc satisfying this condition. No generality is lost in assuming that $\lambda$ contains $p_{n}$ for every $n$, that $\lambda$ has $p$ as one endpoint, and that the sequence $\left\{p_{n}\right\}$ converges monotonically to $p$ on $\lambda$. Let $\lambda=b d p$ where $b$ is the endpoint of $\lambda$ distinct from $p$.

If one of the $\operatorname{arcs} \alpha$ and $\beta$, say $\alpha$, contains a subarc $\alpha_{1}=s t p$ such that $\alpha_{1} \cap \lambda=p$, then $M$ is strongly cyclic at $p$. This contradiction proves (iv).

There exists a positive number $\epsilon$ such that in $S(p, \epsilon)$ no subarc of $\lambda$ containing a point of the sequence $\left\{p_{n}\right\}$ spans one of the arcs $\alpha$ and $\beta$ without intersecting the other. If this is not true, then with the aid of Lemma 2.2 it is seen that $M$ becomes strongly cyclic at $p$. Thus there exists a sequence 
$\left\{\lambda_{n}\right\}$ of distinct arcs converging to $p$ such that $\lambda_{n}=x_{n} y_{n}$ is a subarc of $\lambda$ spanning $J$ and containing a point of the sequence $\left\{p_{n}\right\}, x_{n} \in \alpha^{\prime}, y_{n} \in \beta^{\prime}$, and the sequences $\left\{x_{n}\right\}$ and $\left\{y_{n}\right\}$ converge monotonically to $p$ on $\alpha$ and $\beta$ respectively. Since there is no loss of generality in assuming that $p_{n} \in \lambda_{n}$ for every $n$, this proves (v), (vi), (vii), (viii), and (ix).

To prove (x), assume that $R$ is a component of $M-J$ containing infinitely many of the sets $\left\{\lambda_{i}^{\prime}\right\}$, and hence $p$ is a limit point of $R$. If $p$ is accessible from $R$, it is easily shown that $M$ becomes strongly cyclic at $p$. Thus $p$ is not regularly accessible from $R$. Thus by the lemma of $\mathrm{E}$. E. Betz referred to for the proof of Lemma 2.1 and by Lemma 2.1 there exists a positive number $\epsilon>0$ such that for every positive number $\eta, \eta<\epsilon, R \cap S(p, \eta)$ has an infinite number of components $\left\{B_{i}\right\}$ with $p \in \lim \inf B_{i}, \bar{B}_{i} \cap F[S(p, \eta)] \neq \varnothing$ for every $i$, and for every positive number $\delta$ such that $\delta<\eta, B_{i} \cap S(p, \delta) \neq \varnothing$ for all but a finite number of values of $i$. There exists a value $\eta_{0}$ of $\eta$ such that no component of $R \cap S\left(p, \eta_{0}\right)$ contains more than one element of the collection $\left\{\lambda_{i}^{\prime}\right\}$. If this is not the case then with the aid of Lemma 2.2 it is easily shown that $M$ is strongly cyclic at $p$. Thus it may be assumed that $R \cap S\left(p, \eta_{0}\right)$ has an infinite collection of components $\left\{B_{i}\right\}$ such that $\lambda_{i}^{\prime} \subset B_{i}$ for every $i$. But this makes $M$ strongly cyclic at $p$ since the hypothesis of Lemma 2.3 is satisfied. This contradiction shows that each component of $M-J$ can contain at most a finite number of the sets $\left\{\lambda_{i}^{\prime}\right\}$. Hence it may be assumed that $M-J$ has an infinite collection of components $\left\{R_{i}\right\}$ such that $\lambda_{i}^{\prime} \subset R_{i}$ for every $i$. The proof of $(x)$ is complete if it is observed that if the sequence $\left\{R_{i}\right\}$ does not converge to $p$, then again the hypothesis of Lemma 2.3 is satisfied.

The proof of the theorem is complete if it is noted that if (xi) is not true, then $M$ becomes strongly cyclic at $p$ by an application of Lemma 2.2.

4. The properties $P(r, \alpha)$ and $P(r, \beta)$. In this section let $M$ be a cyclic Peano space, $J=\alpha \cup \beta$ a simple closed curve in $M$ where $\alpha=q x p$ and $\beta=q y p$ are arcs such that $\alpha \cap \beta=q \cup p, q \neq p$, and $r \in M-J$. Let $\alpha^{\prime}=\alpha-(q \cup p)$, $\beta^{\prime}=\beta-(q \cup p)$. The next two theorems are conveniently stated in terms of a set $R(J, r)$ and properties $P(r, \alpha)$ or $P(r, \beta)$ which are defined as follows.

Definition 4.1. By the set $R(J, r)$ is meant the component of $M-J$ containing $r$.

Definition 4.2. The arc $\alpha$ is said to possess the properties $P(r, \alpha)$ with respect to the set $R(J, r)$ provided it possesses both of the following properties:

$P_{1}(r, \alpha):$ No point of $R(J, r)$ separates $r$ from $F[R(J, r)] \cap \alpha^{\prime}$ in $\bar{R}(J, r)-\beta$.

$P_{2}(r, \alpha): F[R(J, r)] \cap \alpha^{\prime}$ is nondegenerate. The properties $P(r, \beta)$ are defined by an interchange of $\alpha$ and $\beta$.

Theorem 4.3 (Spanning Theorem). In order that there exists an arc in $M$ which spans $\alpha$, contains $r$, and is disjoint from $\beta$, it is necessary and sufficient that $\alpha$ possesses the properties $P(r, \alpha)$ with respect to $R(J, r)$. 
Proof. The necessity is immediate. To prove the sufficiency observe that the $\operatorname{arc} \beta$ and the points of $M-\beta$ give an upper semicontinuous decomposition of $M$. Let $M^{\prime}$ be the hyperspace of this decomposition and $f(M)=M^{\prime}$ the associated monotone map. Let $f(\beta)=b$, and note that $f(M-\beta)=M^{\prime}-b$ is one-to-one.

By property $P_{2}(r, \alpha)$ there exist distinct points $z_{1}$ and $z_{2}$ belonging to $\alpha^{\prime} \cap F[R(J, r)]$. Thus $z_{i}$ does not separate $r$ from $z_{j}(i \neq j)$ in $M-\beta$, hence no such separation occurs in $M^{\prime}-b$. By property $P_{1}(r, \alpha)$ no point separates $r$ and $z_{1} \cup z_{2}$ in $M-\beta$, hence no such separation occurs in $M^{\prime}-b$. Thus by a theorem of Hall and Puckett [2, Theorem 2.2, p. 555], $M^{\prime}-b$ contains an arc from $z_{1}$ to $z_{2}$ having $r$ as an interior point. Clearly the inverse under $f$ of this arc is an arc which contains a subarc $a_{1} r a_{2}$ spanning $\alpha^{\prime}$ and such that $a_{1} r a_{2}-\left(a_{1} \cup a_{2}\right) \subset R(J, r)$.

THEOREM 4.4. Let each of the arcs $\alpha$ and $\beta$ possess at most one of the properties $P_{i}(r, \alpha)$ and $P_{i}(r, \beta)$ respectively, $i=1,2$. If there exists an arc $\gamma=$ arb such that $\gamma \cap \alpha^{\prime}=a, \gamma \cap \beta^{\prime}=b$, and $\gamma-(a \cup b) \subset R(J, r)$, then there exists a region $V$ in $R(J, r)$ and two distinct points $c$ and $d$ of the arc $\gamma$ such that the following conditions hold:

(i) $r \in V$.

(ii) $F(V) \supset c \cup d$ where $c$ and $d$ are distinct from $p$ and $q$.

(iii) $F(V) \subset c \cup d \cup q \cup p$.

(iv) $\bar{V}$ is locally connected, hence a Peano space.

Proof. If there exists a region $V$ of $R(J, r)$ satisfying (i), (ii), and (iii), then $\bar{V}$ is locally connected since $\bar{V}-V$ is a finite set.

Since $\alpha$ possesses at most one of the properties $P_{i}(r, \alpha), i=1,2$, the subarc ar of $\boldsymbol{\gamma}$ contains a point $c$ which either separates $r$ from $F[R(J, r)] \cap \alpha^{\prime}$ in $\bar{R}(J, r)-\beta$, or is the single point of $F[R(J, r)] \cap \alpha^{\prime}$. Similarly the subarc $b r$ of $\gamma$ contains a point $d$ which either separates $r$ from $F[R(J, r)] \cap \beta^{\prime}$ in $\bar{R}(J, r)-\alpha$, or is the single point of $F[R(J, r)] \cap \beta^{\prime}$. Thus the component of $R(J, r)-(c \cup d)$ which contains $r$ is the region $V$.

5. Principal theorems. In this section the principal theorems on strongly cyclic Peano spaces are obtained.

Theorem I. In order that a cyclic Peano space $M$ which is strongly arcwise connected at a point $p$ fail to be strongly cyclic at $p$, it is necessary and sufficient that there exists in $M$ a closed set $D$ containing $p$ and a separation $M-D$ $=\left(\cup_{i \in I} V_{i}\right) \cup N$, where $\left\{V_{i}\right\}$ is a collection of distinct components of $M-D$ having the following properties: 1

(i) $V_{i} \rightarrow p$.

(ii) For each $i, F\left(V_{i}\right)=c_{i} \cup d_{i}$ where $c_{i} \cup d_{i} \subset D-p, c_{i} \neq d_{i}$.

(iii) No simple closed curve of $M$ intersects infinitely many of the regions $V_{i}$.

Proof. The sufficiency is immediate. To prove the necessity let $J=\alpha \cup \beta$ 
be any simple closed curve in $M$ containing $p$, where $\alpha=q x p$ and $\beta=q y p$ are arcs such that $\alpha \cap \beta=q \cup p, q \neq p$. Let $\left\{p_{n}\right\}$ and $\left\{\lambda_{n}\right\}$ respectively be the sequences of points and arcs of Theorem 3.1. By Theorem 3.1 there exists an infinite collection of distinct components $\left\{R_{i}\right\}$ of $M-J$ converging to $p$ with $\lambda_{i}^{\prime} \subset R_{i}$. Thus $R_{i}$ is the set $R\left(J, p_{i}\right)$ of Definition 4.1. By Theorems 3.1 (xi) and 4.4 each component $R_{i}$ contains a region $V_{i}$ such that $p_{i} \in V_{i}$, $F\left(V_{i}\right) \subset c_{i} \cup d_{i} \cup q \cup p$, and $F\left(V_{i}\right) \supset c_{i} \cup d_{i}$ where $c_{i} \cup d_{i} \subset \lambda_{i}, c_{i} \neq d_{i}$. Since the sequence $\left\{R_{i}\right\}$ converges to $p$, it may be assumed $q \notin F\left(V_{i}\right)$ for any $i$. If $p \in F\left(V_{k}\right)$ for some integer $k$, then $p$ is accessible from $V_{k}$ by Theorem 4.4 (iv). But this makes $M$ strongly cyclic at $p$ contrary to assumption. Thus it may be assumed that $F\left(V_{i}\right)=c_{i} \cup d_{i}$ for every $i$. The necessity of (i) and (ii) is established by letting $D=p \cup\left[\bigcup_{i \in I}\left(c_{i} \cup d_{i}\right)\right]$.

To prove the necessity of (iii), let $J_{1}$ be a simple closed curve in $M$ containing $p$. If $J_{1} \cap V_{k} \neq \varnothing$ for some integer $k$, then $J_{1}$ contains the points $c_{k}$ and $d_{k}$. If the subarc $c_{k} d_{k}$ of $J_{1}$ lying in $\bar{V}_{k}$ is replaced by the subarc $c_{k} p_{k} d_{k}$ of $\lambda_{k}$, then a simple closed curve containing the points $p_{k}$ and $p$ is obtained. If $J_{1}$ intersects infinitely many of the sets $V_{i}$, then it is seen that $M$ is strongly cyclic at $p$. This contradiction cumpletes the proof of the theorem.

Theorem II. In order that a cyclic Peano space $M$ be strongly cyclic it is necessary and sufficient that for every infinite collection of open sets $\left\{V_{i}\right\}$ in $M$ there exists in $M$ a simple closed curve intersecting infinitely many of these sets.

Proof. The necessity is immediate. To prove the sufficiency, observe that $M$ is strongly arcwise connected. If $M$ is not strongly cyclic, then by Theorem I there is an infinite collection of open sets in $M$ such that no simple closed curve of $M$ intersects infinitely many of these sets. Since this would contradict the hypothesis, it follows that $M$ is strongly cyclic.

Corollary 5.1. If a Peano space $M$ is not separated by any two of its points, then $M$ is strongly cyclic.

Corollary 5.2. If $M$ is a cyclic Peano space which fails to be strongly cyclic at the point $p$, then $p$ is an im kleinen cycle point of $M$.

Theorem 5.3. If $M$ is a cyclic Peano space which is strongly arcwise connected at $p$, but not strongly cyclic at $p$, then given $\epsilon>0$, there exists in $M$ an arc $\gamma=t x p$ having diameter less than $\epsilon$ and an infinite collection of distinct components $\left\{K_{i}\right\}$ of $M-\gamma$ converging to $p$.

Proof. Let $\left\{\alpha_{i}\right\}$ and $\left\{\beta_{i}\right\}$ be the collection of subarcs $\left\{x_{i} x_{i+1}\right\}$ and $\left\{y_{i} y_{i+1}\right\}$ of $\alpha$ and $\beta$ respectively. Let $\alpha_{i}^{\prime}=\alpha_{i}-\left(x_{i} \cup x_{i+1}\right)$. Consider $M-\Gamma$ where $\Gamma=p \cup\left(\bigcup_{i \in I} \lambda_{i}\right) \cup\left(\bigcup_{i \in I} \alpha_{2 i-1}\right) \cup\left(\bigcup_{i \in I} \beta_{2 i}\right)$. If a component $K$ of $M-\Gamma$ should contain infinitely many of the sets $\alpha_{21}^{\prime}$, then $p$ is a limit point of $K$. Since $M$ is not strongly cyclic at $p, p$ is not regularly accessible from $K$. But 
this makes $M$ strongly cyclic at $p$ by applications of Lemmas 2.1 and 2.4. Thus $M-\Gamma$ has an infinite collection $\left\{K_{i}\right\}$ of components each of which contains a set $\alpha_{21}^{\prime}$. Now $K_{i} \rightarrow p$, for otherwise an application of Lemma 2.4 would make $M$ strongly cyclic at $p$. Thus every subarc of $\Gamma$ having $p$ as one endpoint gives the desired separation of $M$, thereby proving the theorem.

6. Two-cyclic Peano spaces. It is known that every Peano space has a basis each element of which is an open connected set having property $S$ $[4$, p. 219]. Thus the closure of an arbitrary element of such a basis is a Peano space. Throughout this section we denote by $\Sigma$ such a basis for the Peano space $M$ under consideration.

Theorem III. Let $M$ be a cyclic Peano space and $p \in M$. Suppose that $M$ is strongly cyclic at $p$, but not two-cyclic at $p$. Then either $p$ belongs to a free arc of $M$ or given $\epsilon>0$, there exists in $M$ an arc $\gamma=t x p$ having diameter less than $\epsilon$ and an infinite collection of distinct components $\left\{K_{i}\right\}$ of $M-\gamma$ converging to $p$.

The proof of Theorem III requires that some background material be developed.

Theorem 6.1. Let $M$ be the Peano space of Theorem III and $U$ an element of $\Sigma$ such that $p \in U$. Then the following conditions hold:

(i) The components of $\bar{U}-p$ may be classified as follows:

(a) $\left\{X_{i}\right\}$ : those components of $\bar{U}-p$ such that $p$ is an endpoint of $\bar{X}_{i}$; or if $p$ is not an endpoint of $\bar{X}_{i}$, then $\bar{X}_{i}$ is not strongly cyclic at $p$.

(b) $\left\{Y_{j}\right\}$ : those components of $\bar{U}-p$ such that $\bar{Y}_{j}$ is strongly cyclic at $p$.

(ii) For each $i$ and $j, \bar{X}_{i} \cap(\bar{U}-U) \neq \varnothing, \bar{Y}_{j} \cap(\bar{U}-U) \neq \varnothing$.

(iii) Each of the collections $\left\{X_{i}\right\}$ and $\left\{Y_{j}\right\}$ is finite.

(iv) If the collection $\left\{X_{i}\right\}$ is nonvacuous, then Theorem III is true.

Proof. Statement (i) is just a classification of the components of $\bar{U}-p$. Statement (ii) follows from the fact that $M$ is cyclic, while (iii) is a consequence of the local connectivity of $M$.

To prove (iv), let $X_{1} \in\left\{X_{i}\right\}$. If $p$ is an endpoint of $\bar{X}_{1}$, Theorem III is easily seen to hold. If $p$ is not an endpoint of $\bar{X}_{1}$ but $\bar{X}_{1}$ is not strongly cyclic at $p$, then Theorem III follows from Theorem 5.3.

In view of Theorem 6.1 (iv), it may be assumed that for the Peano space $M$ of Theorem III the collection $\left\{X_{i}\right\}$ is empty for every element $U$ of $\Sigma$ such that $p \in U$. The space $M$ then possesses the properties which follow:

Property 1. There exists in $M$ an infinite sequence of distinct points $\left\{p_{n}\right\}$ converging to $p$ such that if $\Gamma_{1}$ and $\Gamma_{2}$ are two arcs of $M$ having $p$ as one endpoint and each containing infinitely many of the points $\left\{p_{n}\right\}$, then $\Gamma_{1} \cap \Gamma_{2}$ consists of a set of points having $p$ as a limit point. Moreover, there exists in $M$ an arc $\alpha$ $=q x p$ such that the points $\left\{p_{n}\right\}$ converge monotonically to $p$ on $\alpha$.

Property 2. If $U \in \Sigma$ and $p \in U$, then all but a finite number of the points 
$\left\{p_{n}\right\}$ of property 1 lie in a true cyclic element $E$ of the set $\bar{Y}_{1}$ where $\bar{U}-p$ $=\mathrm{U}_{j=1}^{h} Y_{j}$.

Proof. Let $U \in \Sigma$ such that $p \in U$. Then $\bar{U}-p=\bigcup_{j=1}^{h} Y_{j}$. In view of Property 1 it may be assumed that all but a finite number of the points $\left\{p_{n}\right\}$ lie in $Y_{1}$. Let $E$ be the true cyclic element of $\bar{Y}_{1}$ containing $p$. Since $M$ is cyclic, every component of $\bar{Y}_{1}-E$ must intersect $\bar{U}-U$, hence the components of $\bar{Y}_{1}-E$ cannot have limit points in $E$ arbitrarily close to $p$. Thus all but a finite number of the points $\left\{p_{n}\right\}$ lie in $E$.

Property 3. $E-\alpha=H \cup N$, where $H$ is the set of all points $x \in E$ for which there exists in $E$ a nondegenerate arc $x p$ with $x p \cap \alpha=p, N$ is the set of all points $x \in E$ for which no such arc exists.

PROPERTy 4. If, for every component $W$ of $E-\alpha, \bar{W} \cap F(M-E) \neq \varnothing$, then $p$ is not a limit point of $N$ and the collection $\left\{C_{i}\right\}$ of components of $H$ is finite. Thus there exists a subarc op of $\alpha$ which does not separate $M$. Moreover, in this case there exists at least one component of $H$ which has a set of limit points in $\alpha-p$ having $p$ as a limit point.

Proof. By the definition of $N, p$ is not accessible from $N$. Thus if $p$ is a limit point of $N$, the hypothesis of Lemma 2.4 is easily seen to be satisfied. Since this makes $M$ two-cyclic at $p, p$ is not a limit point of $N$. If $H$ has an infinite collection of components, the hypothesis of Lemma 2.4 is again satisfied. Thus $H$ has a finite number of components in this case. In the natural order from $q$ to $p$ on $\alpha$, let $d$ be the last point of $\bar{N} \cap \alpha$. Thus for any point $v$ interior to the subarc $d p$, the subarc $v p$ of $\alpha$ does not separate $M$. The proof of Property 4 is complete if it is observed that if $H$ does not have a component which has a set of limit points in $\alpha-p$ having $p$ as a limit point, then there is an element $V \in \Sigma$ containing $p$ for which the collection $\left\{X_{i}\right\}$ is nonempty.

PROPERTy 5. If, for arbitrarily small elements $U$ of $\Sigma$ such that $p \in U$ there exists a component $W$ of $E-\alpha$ such that $\bar{W} \cap F(M-E)=\varnothing$, then Theorem III is true.

The following restrictions may be imposed on all further discussion.

Restrictions 6.2. The space $M$ under consideration is a cyclic Peano space which is strongly cyclic at the point $p$, but not two-cyclic at $p$, and for which every element $U$ of the basis $\Sigma$ such that $p \in U$ satisfies the following conditions:

(a) The collection $\left\{X_{i}\right\}$ is empty.

(b) For every component $W$ of $E-\alpha, \bar{W} \cap F(M-E) \neq \varnothing$, where $E$ and $W$ are the sets of $\bar{U}$ as given in the above properties.

Theorem 6.3. Let $M$ be a Peano space satisfying the Restrictions 6.2. Then there exists a sequence $\left\{U_{i}\right\}$ of elements of the basis $\Sigma$ such that $p \in U_{i}$ for every $i$, and corresponding sequences $\left\{E_{i}\right\},\left\{N_{i}\right\}$, and $\left\{H_{i}\right\}$ as given by Properties 2 and 3, such that the following conditions hold: 
(i) $\left\{U_{i}\right\}$ is a null sequence, $U_{i} \supset \bar{U}_{i+1}$, and $\bar{U}_{i+1} \cap N_{i}=\varnothing$.

(ii) $E_{i} \supset E_{i+1}$ and $H_{i} \supset \vec{H}_{i+1}$.

(iii) If $R_{i}$ is a component of $H_{i}$ having a set of limit points in $\alpha-p$ which has $p$ as a limit point, then there exists a sequence $\left\{R_{j}\right\}, j \geqq i$, such that $R_{j}$ is a component of $H_{j}$ having a set of limit points in $\alpha-p$ which has $p$ as a limit point, and $R_{j} \supset \bar{R}_{j+1}$.

Proof. The sequences $\left\{U_{i}\right\},\left\{E_{i}\right\},\left\{N_{i}\right\}$, and $\left\{H_{i}\right\}$ are easily seen to exist. To prove (iii), note that by Property 4 each set $H_{i}$ contains at least one component having a set of limit points in $\alpha-p$ having $p$ as a limit point. Let $R_{1}$ be such a component of $H_{1}$. In the natural order from $q$ to $p$ on $\alpha$ let $d$ be the last point of $\bar{N}_{1} \cap \alpha$. There exists a sequence $\left\{r_{n}\right\}$ of points interior to the subarc $d p$ of $\alpha$ such that $r_{n} \rightarrow p, r_{n}$ is a limit point of $R_{1}$, and $r_{n} \in U_{2}$, $n \in I$. Since $H_{2}$ has only a finite number of components, it is clear that there is a component $R_{2}$ of $H_{2}$ which has infinitely many of the points $\left\{r_{n}\right\}$ as limit points and $\bar{R}_{2} \subset R_{1}$. Continuing this process inductively completes the proof of the theorem.

Theorem 6.4. For each sequence $\left\{R_{j}\right\}, j \geqq i$, of Theorem 6.3(iii) there exists an arc $\lambda$ from a point $t_{i} \in F\left(R_{i}\right)-\alpha$ to $p$, and a sequence $\left\{\mu_{j}\right\}$ of arcs such that

(a) $\lambda-p \subset \bar{R}_{i}-\alpha$; i.e., $\lambda \cap \alpha=p$.

(b) $\lambda \cap R_{j} \neq \varnothing$ for every $j \geqq i$.

(c) $\mu_{j}=x_{j} y_{j}$ is an arc of $E_{j}-E_{j+1}$ such that $\mu_{j} \cap \alpha=x_{j}, \mu_{j} \cap \lambda=y_{j}$.

(d) The sequences $\left\{x_{j}\right\}$ and $\left\{y_{j}\right\}$ converge monotonically to $p$ on $\alpha$ and $\lambda$ respectively.

Proof. An arc $\lambda$ satisfying (a) and (b) is readily constructed. In each set $E_{i}$, let $t_{i} \in \alpha, z_{i} \in \lambda$. There exists an arc $p z_{i} t_{i}$ in $E_{i}$, and this arc contains a subarc $\mu_{i}=x_{i} y_{i}$ such that $\mu_{i} \cap \alpha=x_{i}, \mu_{i} \cap \lambda=y_{i}$. It is clear that no generality is lost in assuming that (c) and (d) hold.

THEOREM 6.5. Each set $H_{i}, i \in I$, of Theorem 6.3 contains exactly one component $R_{i}$ having a set of limit points in $\alpha-p$ which has $p$ as a limit point. Thus the sequence $\left\{R_{i}\right\}, i \in I$, of Theorem 6.3(iii) is unique, as is the sequence $\left\{G_{i}\right\}$ where $G_{i}$ is the set of all points $x \in R_{i}$ which can be joined to $\alpha-p$ by an arc of $E_{i}$ not meeting $\lambda$.

Proof. Assume that there is a set $H_{i}$ which has two components having a set of limit points in $\alpha-p$ each having $p$ as a limit point. It may be assumed that $H_{1}$ is such a set. By Theorem 6.3(iii) there exist two distinct sequences $\left\{R_{i}\right\}$ and $\left\{S_{i}\right\}$ as there described. As described in Theorem 6.4, there exist $\operatorname{arcs} \lambda$ and $\left\{\mu_{i}\right\}$ for the sequence $\left\{R_{i}\right\}$, and $\operatorname{arcs} \lambda^{*}$ and $\left\{\mu_{i}^{*}\right\}$ for the sequence $\left\{S_{i}\right\}$. It is easily seen that this makes $M$ two-cyclic at $p$. This contradiction proves the theorem.

A review of Properties 1-5 and Theorems 6.3-6.5 reveals that there is no loss of generality in making the following assumptions. 
Assumptions 6.6. For the Peano space $M$ of Properties $1-5$ and Theorems 6.3-6.5 the following conditions hold:

(a) In the natural order from $q$ to $p$ on $\alpha, x_{i}<p_{i}<x_{i+1}, i \in I$.

(b) $p_{i} \in E_{i}-E_{i+1}, i \in I$.

(c) $\mu_{i} \subset E_{i}-E_{i+1}$.

(d) $\lambda$ is an arc from a point $t \in F\left(R_{1}\right)-\alpha$ to the point $p$ such that $\lambda$ satisfies Theorem 6.4 for the sequence $\left\{R_{i}\right\}$.

Theorem 6.7. Let $M$ be a Peano space satisfying the restrictions 6.2 and subject to the Assumptions 6.6. Let $\left\{\alpha_{i}\right\}$ be the collection of subarcs of $\alpha$ defined by $\alpha_{i}=x_{i} p_{i} x_{i+1},\left\{\lambda_{i}\right\}$ the collection of subarcs of $\lambda$ defined by $\lambda_{i}=y_{i} y_{i+1}$. Let $\alpha_{i}^{\prime}=\alpha_{i}-\left(x_{i} \cup x_{i+1}\right), \lambda_{i}^{\prime}=\lambda_{i}-\left(y_{i} \cup y_{i+1}\right)$. For each set $G_{j}$ of the sequence $\left\{G_{i}\right\}$ of sets defined in Theorem 6.5, consider the set $G_{j}-\bigcup_{i \in I} \mu_{i}, j \in I$. Then there exists a positive integer $k$ such that for $j \geqq k$ the following hold:

(i) No component of $G_{j}-\bigcup_{i \in I} \mu_{i}$ has limit points in two distinct sets $\alpha_{m}^{\prime}$ and $\alpha_{n}^{\prime}$ where $|m-n| \geqq 2$.

(ii) If a component of $G_{j}-\bigcup_{i \in I} \mu_{i}$ has limit points in a set $\alpha_{m}^{\prime}$ and a set $\lambda_{n}^{\prime}$, then $m=n$.

(iii) If a component of $G_{j}-\bigcup_{i \in I} \mu_{i}$ has limit points in a set $\alpha_{m}^{\prime}$, then any limit points of this component in $\bigcup_{i \in I} \mu_{i}^{\prime}$, where $\mu_{i}^{\prime}=\mu_{i}-\left(x_{i} \cup y_{i}\right)$, must lie in $\mu_{m}^{\prime} \cup \mu_{m+1}^{\prime}$.

Proof. If no such integer $k$ exists, it is easy to construct two arcs $\Gamma_{1}$ and $\Gamma_{2}$ such that $\Gamma_{1} \cap \Gamma_{2}=p$ and each contains infinitely many of the points $\left\{p_{n}\right\}$.

Proof of Theorem III. Theorem 6.1 (iv) and property 5 give special cases in which Theorem III is true. It may thus be assumed that $M$ satisfies the Restrictions 6.2 and is subject to the Assumptions 6.6. Moreover, it may be assumed in Theorem 6.7 that the integer $k=1$. In the natural order from $q$ to $p$ on $\alpha$, let $d$ be the last point of $\bar{N}_{1} \cap \alpha$. Then there exists an integer $r$ such that for $i \geqq r, x_{i}$ is a point interior to the subarc $d p$ of $\alpha$. No generality is lost in assuming that $r=1$. Define an arc $\Gamma$ as follows:

$$
\Gamma=p \cup\left(\bigcup_{i \in I} \alpha_{2 i-1}\right) \cup\left(\bigcup_{i \in I} \mu_{i}\right) \cup\left(\bigcup_{i \in I} \lambda_{2 i}\right) \text {. }
$$

Note that the arc $\Gamma$ lies in the set $E_{1}$ and is disjoint from $\bar{N}_{1}$.

Next define a collection of sets $\left\{K_{i}\right\}$ as follows:

$K_{i}=\alpha_{2 i}^{\prime} \cup\left(\right.$ all components of $G_{1}-\bigcup_{i \in I} \mu_{i}$ having limit points in $\alpha_{2 i}^{\prime}$ ).

Observe that each set $K_{i}$ is a component of $E_{1}-\Gamma$ by Theorem 6.7. Thus $E_{1}$ is separated into infinitely many components $\left\{K_{i}\right\}$ by the arc $\Gamma$. Moreover, the $\left\{K_{i}\right\}$ converge to $p$, for otherwise the hypothesis of Lemma 2.4 is satisfied thereby making $E_{1}$, and hence $M$, two-cyclic at $p$. Thus $\Gamma$, and every subarc of $\Gamma$ having $p$ as an endpoint, separates $M$ in to infinitely many components converging to $p$, the collection of components in such cases being a 
G. R. STROHL, JR.

subcollection of the collection $\left\{K_{i}\right\}$. This completes the proof of Theorem III.

\section{BIBLIOGRAPHY}

1. D. W. Hall and W. T. Puckett, Jr., Conditions for the continuity of arc-preserving transformations, Bull. Amer. Math. Soc. vol. 47 (1941) pp. 468-475.

2. - Strongly arcwise connected spaces, Amer. J. Math. vol. 63 (1941) pp. 554-562.

3. E. E. Betz, Accessibility and separation by simple closed curves, Amer. J. Math. vol. 63 (1941) pp. 127-135.

4. D. W. Hall and G. L. Spencer, II, Elementary topology, New York, John Wiley \& Sons, 1955.

5. G. T. Whyburn, Analytic topology, New York, Amer. Math. Soc. Colloquium Publications, vol. 28, 1942.

UNIVERSITY OF MARYLAND,

College Park, Md.

United States Naval Academy,

AnNAPOLIS, MD. 